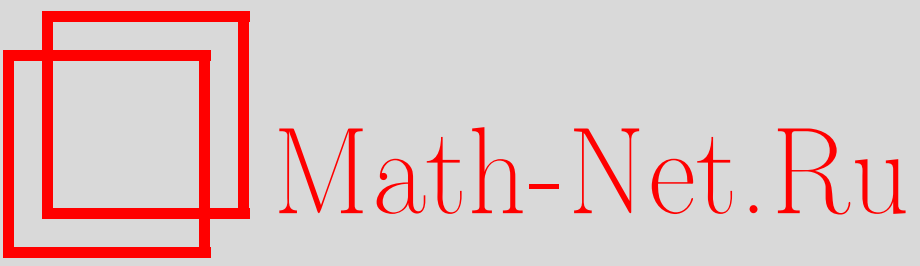

О. В. Камловский, Нелинейность одного класса булевых функций, построенных с использованием двоичных разрядных последовательностей линейных рекуррент над кольцом $\mathbb{Z}_{2^{n}}$, Матем. вопр. криптогр., 2016, том 7, выпуск 3, 29-46

DOI: https://doi.org/10.4213/mvk194

Использование Общероссийского математического портала Math-Net.Ru подразумевает, что вы прочитали и согласны с пользовательским соглашением

http://www.mathnet.ru/rus/agreement

Параметры загрузки:

IP : 18.207 .199 .55

26 апреля 2023 г., 04:56:30 
МАТЕМАТИЧЕСКИЕ ВОПРОСЫ КРИПТОГРАФИИ

2016 T. 7 № 3 C. $29-46$

УДК 519.113.6+519.719.2

\title{
Нелинейность одного класса булевых функций, построенных с использованием двоичных разрядных последовательностей линейных рекуррент над кольцом $\mathbb{Z}_{2^{n}}$ \\ О. В. Камловский
}

ООО «Центр сертификационных исследований», Москва

Получено 30.V.2016

\begin{abstract}
Аннотация. В работе с использованием двоичных разрядных последовательностей линейных рекуррент над кольцом вычетов по модулю $2^{n}$ строится класс булевых функций. Для этого класса функций получены оценки снизу их коэффициентов нелинейности.

Ключевые слова: булевы функции, коэффициенты Уолша-Адамара, нелинейность, линейные рекуррентные последовательности

Nonlinearity of a class of Boolean functions constructed using significant bits of linear recurrences over the ring $\mathbb{Z}_{2^{n}}$

O. V. Kamlovskiy

Sertification Research Center, LLC, Moscow
\end{abstract}

\begin{abstract}
We construct a class of Boolean functions defined by the significant bits of linear recurrent sequences over the ring $\mathbb{Z}_{2^{n}}$. For this class of functions bounds for nonlinearity coefficients are obtained.
\end{abstract}

Keywords: Boolean functions, Walsh coefficients, nonlinearity, linear recurrent sequences

Citation: Mathematical Aspects of Cryptography, 2016, v. 7, № 3, pp. 29-46 (Russian)

(C) Академия криптографии Российской Федерации, 2016 г. 


\section{1. Введение}

Пусть $R=\mathbb{Z}_{2^{n}}=\left\{0,1, \ldots, 2^{n}-1\right\}-$ кольцо вычетов по модулю $2^{n}$. Для каждого элемента $a \in R$ справедливо двоичное разложение

$$
a=a_{0}+2 a_{1}+2^{2} a_{2}+\cdots+2^{n-1} a_{n-1},
$$

где $a_{i} \in\{0,1\}, i=0,1, \ldots, n-1$. Рассмотрим отображения $f: R \rightarrow\{0,1\}$, построенные по правилу

$$
f(a)=a_{n-1} \oplus a_{n-t_{1}} \oplus a_{n-t_{2}} \oplus \cdots \oplus a_{n-t_{k}}, \quad a \in R,
$$

где $0 \leqslant k \leqslant n-1,2 \leqslant t_{1}<t_{2}<\cdots<t_{k} \leqslant n, a_{n-1}, a_{n-t_{1}}, a_{n-t_{2}}, \ldots, a_{n-t_{k}}-$ соответствующие двоичные разряды числа $a$.

Выберем произвольный примитивный многочлен $F(x)$ степени $m$ над полем $\mathbb{Z}_{2}$ (см. [1-2]). Для его периода справедливо равенство $T(F)=2^{m}-1$. Рассмотрим отмеченный многочлен $G(x) \in R[x]$, соответствующий многочлену $F(x)$, т. е. многочлен, удовлетворяющий двум следующим условиям:

1) многочлен, полученный из $G(x)$ приведением всех его коэффициентов по модулю 2, совпадает с $F(x)$;

2) $T(G)=T(F)$.

А. А. Нечаев [3] показал, что такой многочлен $G(x)$ существует и что он единствен, причем имеется довольно простой рекурсивный способ его построения по многочлену $F(x)$.

Пусть $v$ - такая линейная рекуррентная последовательность (ЛРП) порядка $m$ над кольцом $R$ с характеристическим многочленом $G(x)$, что последовательность $\bar{v}$, полученная из $v$ приведением всех ее элементов по модулю 2 , является ненулевой. Рассмотрим булеву функцию $g=g_{v, f}$ от $m$ переменных, определенную по правилу: $g(0,0, \ldots, 0)=0$ и для всех $i=0,1, \ldots, 2^{m}-2$

$$
g(\bar{v}(i), \bar{v}(i+1), \ldots, \bar{v}(i+m-1))=f(v(i)) .
$$

Последовательность $\bar{v}$ является ЛРП максимального периода над полем $\mathbb{Z}_{2}$, поэтому среди элементов $(\bar{v}(i), \bar{v}(i+1), \ldots, \bar{v}(i+m-1)), \quad i=$ $0,1, \ldots, 2^{m}-2$, появляются по одному разу все ненулевые двоичные векторы длины $m$. Таким образом, правило (2) однозначно задает булеву функцию $g$. Таблицу данной функции легко построить с использованием линейного регистра сдвига над кольцом $R$, вырабатывающего последовательность $v$ в качестве выходной последовательности. 
Нелинейностью $\mathrm{nl}(g)$ булевой функции $g$ (см., например, [4]) называется расстояние Хэмминга от булевой функции $g$ до класса всех аффинных булевых функций от $m$ переменных. Для произвольной булевой функции $g$ от $m$ переменных верна оценка

$$
\operatorname{nl}(g) \leqslant 2^{m-1}-2^{\frac{m}{2}-1}
$$

которая достижима только для бент-функций.

В [5] был получен следующий результат. Пусть $n>1$ и $f(a)=a_{n-1} \oplus$ $\eta\left(a_{0}, a_{1}, \ldots, a_{n-2}\right)$, где $\eta:\{0,1\}^{n-1} \rightarrow\{0,1\}-$ произвольное отображение, тогда для нелинейности булевой функции $g$, построенной по правилу (2), справедлива оценка

$$
\mathrm{nl}(g) \geqslant 2^{m-1}-\left(2^{n-1}-1\right) 2^{\frac{m+n-3}{2}} .
$$

Таким образом, $\operatorname{nl}(g) \geqslant 2^{m-1}-h_{1}(n) 2^{m / 2}$, где $h_{1}(n)=O\left(2^{3 n / 2}\right)$ при $n \rightarrow \infty$.

Параметр $n$ существенно влияет на степень $\operatorname{deg} g$ многочлена Жегалкина рассматриваемых функций. Так, в [6] показано, что при тождественно нулевой функции $\eta$ и $m>2^{n}$ выполнено равенство $\operatorname{deg} g=2^{n-1}$.

Представляет интерес уточнение оценки (3) для класса отображений $f$ вида (1). В этой работе показано, что если $f(a)=a_{n-1} \oplus a_{n-t}$ или $f(a)=$ $a_{n-1} \oplus a_{n-t+1} \oplus a_{n-t}$, то

$$
\mathrm{nl}(g) \geqslant 2^{m-1}-\left(2^{n-1}-1\right) 2^{\frac{m}{2}-1}\left(\frac{2}{\pi} \ln \left(2^{n-1}\right)+1\right)\left(\frac{2}{\pi} \ln \left(2^{t-2}\right)+1\right) .
$$

Таким образом, для функций $g=g_{v, f}$, соответствующих данным отображениям, имеет место неравенство $\mathrm{nl}(g) \geqslant 2^{m-1}-h_{2}(n) 2^{m / 2}$, где $h_{2}(n)=O\left(n 2^{n}\right)$ при $n \rightarrow \infty$.

Отметим, что с помощью линейных рекуррент над полями удается строить булевы функции, для которых гарантируются значительно худшие значения нелинейности (теоретические обоснования того, какие они на самом деле, отсутствуют). Например, в [7] построены булевы функции $g$ от $m$ переменных с нелинейностью

$$
\operatorname{nl}(g) \geqslant 2^{m-1}-h_{3}(m) 2^{\frac{m}{2}},
$$

где $h_{3}(m)=O\left(2^{3 m / 8} m^{1 / 4}\right)$ при $m \rightarrow \infty$. 


\section{2. Суммы модулей спектральных коэффициентов}

Рассмотрим произвольное отображение $f: R \rightarrow \mathbb{Z}_{2}=\{0,1\}$. Пусть $\chi$ есть характер группы $\left(\mathbb{Z}_{2^{n}},+\right)$, определенный равенством $\chi(x)=e^{2 \pi i x / 2^{n}}$, $x \in \mathbb{Z}_{2^{n}}$. Множество всех характеров рассматриваемой группы имеет вид $\left\{\chi_{a}: a \in \mathbb{Z}_{2^{n}}\right\}$, где $\chi_{a}(x)=\chi(a x), \quad x \in \mathbb{Z}_{2^{n}}$. Согласно результатам работ [8-9] найдутся однозначно определенные комплексные числа $\nu_{j}=\nu_{j}(f)$, для которых

$$
(-1)^{f(a)}=\sum_{j=0}^{2^{n}-1} \nu_{j} \chi(a j), \quad a=0,1, \ldots, 2^{n}-1 .
$$

Числа $\nu_{j}$ будем называть спектральными коэффициентами. Они вычисляются по формуле

$$
\nu_{j}=\frac{1}{2^{n}} \sum_{s=0}^{2^{n}-1}(-1)^{f(s)} \chi(-s j), \quad j=0,1, \ldots, 2^{n}-1 .
$$

Введем обозначение

$$
\sigma(f)=\sum_{j=0}^{2^{n}-1}\left|\nu_{j}\right|
$$

В [5] показано, что

$$
\mathrm{nl}(g) \geqslant \sigma(f)\left(2^{n-1}-1\right) 2^{\frac{m}{2}-1} .
$$

Изучим параметр $\sigma(f)$ для отображений $f$ вида (1).

Теорема 1 ([10]). Если в равенстве (1) $k=0$, m.е. $f(a)=a_{n-1}$, то

$$
\left|\nu_{j}\right|= \begin{cases}0, & \text { если } j-\text { четное число } \\ \frac{1}{2^{n-1}} \frac{1}{\sin \frac{\pi j}{2^{n}}}, & \text { если } j-\text { нечетное число. }\end{cases}
$$

При этом для величиньь

$$
\sigma_{n}=\frac{1}{2^{n-1}} \sum_{j=1,3, \ldots, 2^{n}-1} \frac{1}{\sin \frac{\pi j}{2^{n}}}=\frac{1}{2^{n-2}} \sum_{j=1,3, \ldots, 2^{n-1}-1} \frac{1}{\sin \frac{\pi j}{2^{n}}}
$$

справедлива оценка

$$
\sigma_{n} \leqslant \frac{2}{\pi} \ln \left(2^{n-1}\right)+1
$$


Величина $\delta_{n}=\frac{2}{\pi} \ln \left(2^{n-1}\right)+1$, указанная в теореме 1 , линейна по параметру $n$. Вопрос о точности оценки $\sigma_{n} \leqslant \delta_{n}$ остается пока открытым. Приведем таблицу нескольких первых значений величин $\sigma_{n}$ и $\delta_{n}$ (с точностью до трех десятичных знаков после запятой), полученных с использованием ЭВМ:

\begin{tabular}{|c|c|c|c|c|c|c|c|c|c|c|c|}
\hline$n$ & 1 & 2 & 3 & 4 & 5 & 6 & 7 & 8 & 9 & 10 & 11 \\
\hline$\sigma_{n}$ & 1 & 1,414 & 1,847 & 2,287 & 2,727 & 3,168 & 3,610 & 4,051 & 4,492 & 4,933 & 5,375 \\
\hline$\delta_{n}$ & 1 & 1,441 & 1,882 & 2,323 & 2,765 & 3,206 & 3,647 & 4,088 & 4,530 & 4,971 & 5,412 \\
\hline
\end{tabular}

\begin{tabular}{|c|c|c|c|c|c|c|c|c|c|c|}
\hline$n$ & 12 & 13 & 14 & 15 & 16 & 17 & 18 & 19 & 20 & 21 \\
\hline$\sigma_{n}$ & 5,816 & 6,257 & 6,699 & 7,140 & 7,581 & 8,022 & 8,464 & 8,905 & 9,346 & 9,787 \\
\hline$\delta_{n}$ & 5,853 & 6,295 & 6,736 & 7,177 & 7,619 & 8,060 & 8,501 & 8,942 & 9,384 & 9,825 \\
\hline
\end{tabular}

\begin{tabular}{|c|c|c|c|c|c|c|c|c|c|}
\hline$n$ & 22 & 23 & 24 & 25 & 26 & 27 & 28 & 29 & 30 \\
\hline$\sigma_{n}$ & 10,229 & 10,670 & 11,111 & 11,553 & 11,994 & 12,435 & 12,876 & 13,318 & 13,759 \\
\hline$\delta_{n}$ & 10,266 & 10,707 & 11,149 & 11,590 & 12,031 & 12,473 & 12,914 & 13,355 & 13,796 \\
\hline
\end{tabular}

Эта таблица показывает, что для рассмотренных значений $n$ приведенная оценка является достаточно точной.

В дальнейшем мы используем следующие леммы.

Лемма 1. Пусть $2 \leqslant t \leqslant n, f(a)=h(a) \oplus a_{n-t}, a \in \mathbb{Z}_{2^{n}}$, где отображение $h: \mathbb{Z}_{2^{n}} \rightarrow \mathbb{Z}_{2}$ фиктивно зависит от разряда $a_{n-t}$. Тогда для всех таких $j=0,1, \ldots, 2^{n}-1$, что $j \neq 2^{t-1}+2^{t} s$, где $s \in \mathbb{Z}$,

$$
\left|\nu_{j}(f)\right|=\left|\nu_{j}(h)\right| \frac{\left|1-e^{-2 \pi i j / 2^{t}}\right|}{\left|1+e^{-2 \pi i j / 2^{t}}\right|} .
$$

Доказательство. Из равенства (1) с использованием двоичного разложения $s=s_{0}+2 s_{1}+2^{2} s_{2}+\ldots+2^{n-1} s_{n-1}$ получим

$$
\begin{aligned}
& \nu_{j}(f)= \\
& =\frac{1}{2^{n}} \sum_{s_{0}, \ldots, s_{n-1} \in\{0,1\}}(-1)^{\widetilde{h}\left(s_{0}, \ldots, s_{n-1}\right) \oplus s_{n-t}} \exp \left\{-2 \pi i \frac{\left(s_{0}+\cdots+2^{n-1} s_{n-1}\right) j}{2^{n}}\right\},
\end{aligned}
$$

где $\widetilde{h}$ - такая булева функция от $n$ переменных, что

$$
\widetilde{h}\left(a_{0}, \ldots, a_{n-1}\right)=h(a)
$$

для любого $a \in \mathbb{Z}_{2^{n}}$. 
В силу фиктивности зависимости $\widetilde{h}$ от $a_{n-t}$

$$
\nu_{j}(f)=S_{j} \sum_{s_{n-t} \in\{0,1\}}(-1)^{s_{n-t}} e^{-2 \pi i s_{n-t} j / 2^{t}}
$$

где

$$
\begin{aligned}
& S_{j}= \\
& =\frac{1}{2^{n}} \sum_{\substack{i \in\{\in\{0,1\}: \\
i \in\{0, \ldots, n-1\} \backslash\{n-t\}}}(-1)^{\widetilde{h}\left(s_{0}, \ldots, s_{n-t-1}, 0, s_{n-t+1}, \ldots, s_{n-1}\right)} \exp \left\{-2 \pi i \frac{\sum_{i \neq n-t} 2^{i} s_{i}}{2^{n}} j\right\} .
\end{aligned}
$$

Значит,

$$
\left|\nu_{j}(f)\right|=\left|S_{j}\right|\left|1-e^{-2 \pi i j / 2^{t}}\right| .
$$

С другой стороны, согласно равенству (5)

$$
\begin{aligned}
& \nu_{j}(h)=\sum_{=\frac{1}{2^{n}}} \sum_{s_{0}, \ldots, s_{n-1} \in\{0,1\}}(-1)^{\widetilde{h}\left(s_{0}, \ldots, s_{n-1}\right)} \exp \left\{-2 \pi i j \frac{s_{0}+\cdots+2^{n-1} s_{n-1}}{2^{n}}\right\}= \\
& =\frac{1}{2^{n}} \sum_{\substack{i \in\{0, \ldots, n-1\} \backslash\{n-t\} \\
s_{i} \in\{0,1\}:}}(-1)^{\widetilde{h}\left(s_{0}, \ldots, s_{n-t-1}, 0, s_{n-t+1}, \ldots, s_{n-1}\right)} \times \\
& \quad \times \exp \left\{-2 \pi i \frac{\sum_{i \neq n-t} 2^{i} s_{i}}{2^{n}} j\right\} \sum_{s_{n-t} \in\{0,1\}}(-1)^{\widetilde{h}\left(s_{0}, \ldots, s_{n-1}\right)} e^{-2 \pi i s_{n-t} j / 2^{t}}
\end{aligned}
$$

а следовательно,

$$
\left|\nu_{j}(h)\right|=\left|S_{j}\right|\left|1+e^{-2 \pi i j / 2^{t}}\right| .
$$

Заметим, что $1+e^{-2 \pi i j / 2^{t}} \neq 0$ при рассматриваемых значениях $j$, поэтому из равенств (8) и (9) следует требуемый результат.

Лемма 2. Пусть отображение $f$ задано формулой (1), тогда для всех $j=$ $0,2,4, \ldots, 2^{n}-2$ имеет место равенство $\nu_{j}(f)=0$.

Доказательство. Соотношения непосредственно следуют из [11, предложение 2]. 
Теорема 2. Если отображение $f$ задано равенством (1), то

$$
\left|\nu_{j}(f)\right|= \begin{cases}0, & \text { если } j-\text { четное число }, \\ \frac{1}{2^{n-1}} \frac{1}{\sin \frac{\pi j}{2^{n}}}\left|\prod_{l=1}^{k} \operatorname{tg} \frac{\pi j}{2^{t_{l}}}\right|, & \text { если } j-\text { нечетное число. }\end{cases}
$$

Доказательство. Достаточно применить метод математической индукции по параметру $k$, опираясь на теорему 1 , леммы 1,2 и равенство

$$
\frac{\left|1-e^{i \varphi}\right|}{\left|1+e^{i \varphi}\right|}=\left|\operatorname{tg} \frac{\varphi}{2}\right|
$$

3. Функция $f(a)=a_{n-1} \oplus a_{n-t}$

Рассмотрим отображение $f(a)=a_{n-1} \oplus a_{n-t}$, в котором $2 \leqslant t \leqslant n, a=$ $a_{0}+2 a_{1}+2^{2} a_{2}+\ldots+2^{n-1} a_{n-1} \in \mathbb{Z}_{2^{n}}, a_{0}, a_{1}, \ldots, a_{n-1} \in\{0,1\}$. По теореме 2 для спектральных коэффициентов $\nu_{j}=\nu_{j}(f)$ справедливо соотношение

$$
\left|\nu_{j}\right|= \begin{cases}0, & \text { если } j-\text { четное число, } \\ \frac{1}{2^{n-1}} \frac{1}{\sin \frac{\pi j}{2^{n}}}\left|\operatorname{tg} \frac{\pi j}{2^{t}}\right|, & \text { если } j-\text { нечетное число. }\end{cases}
$$

Утверждение 1. Пусть $c_{t j}=\left|\operatorname{tg} \frac{\pi j}{2^{t}}\right|$, где $j=1,3, \ldots, 2^{n}-1$. Тогда

1) $c_{t j}=c_{t 2^{t}+j}$ для всех таких $j$, что $2^{t}+j \leqslant 2^{n-1}$,

2) $c_{t 2^{t-1}-j}=c_{t 2^{t-1}+j}$ для всех таких $j$, что $2^{t-1}+j \leqslant 2^{n-1}$ u $2^{t-1}-j \geqslant 1$,

3) числа $c_{t j}$ монотонно возрастают при всех $j \leqslant 2^{t-1}-1$,

4) справедливо равенство $\left(c_{t 1}+c_{t 3}+c_{t 5}+\cdots+c_{t 2^{t-1}-1}\right) / 2^{t-2}=\sigma_{t-1}$, где величина $\sigma_{t-1}$ определена равенством (7),

5) при $t \geqslant 3$ существует такое единственное число $s \in\{1,3,5, \ldots$ $\left.\ldots, 2^{t-1}-3\right\}$, что

$c_{t 1} \leqslant \sigma_{t-1}, \ldots, c_{t 2^{t-1}-s-2} \leqslant \sigma_{t-1}, \quad c_{t 2^{t-1}-s}>\sigma_{t-1}, \ldots, c_{t 2^{t-1}-1}>\sigma_{t-1}$. 
Доказательство. Утверждения пп. 1-3 очевидны. Для доказательства п. 4 достаточно заметить, что

$$
\frac{1}{2^{t-2}} \sum_{j \in\left\{1,3, \ldots, 2^{t-1}-1\right\}} c_{t j}=\frac{1}{2^{t-2}} \sum_{j \in\left\{1,3, \ldots, 2^{t-1}-2\right\}}\left(c_{t j}+c_{t 2^{t-1}-j}\right)
$$

и

$$
c_{t j}+c_{t 2^{t-1}-j}=\frac{2}{\sin \frac{\pi j}{2^{t-1}}} .
$$

Утверждение п. 5 непосредственно следует из пп. 3 и 4.

Лемма $3([12])$. Если функиия $h(x)$ выпукла вниз на отрезке $[a, b]$, то для всех таких $x, y \in[a, b]$ u $r \in \mathbb{R}$, что $x-r, y+r \in[a, b]$, выполнено неравенство $h(x-r)+h(y-r) \geqslant h(x)+h(y)$.

Утверждение 2. Если $f(a)=a_{n-1} \oplus a_{n-t}$, где $2 \leqslant t \leqslant n$, то

$$
\sigma(f)=\sum_{j=0}^{2^{n}-1}\left|\nu_{j}\right| \leqslant \sigma_{n} \sigma_{t-1} .
$$

В частности, $\sigma(f) \leqslant\left(\frac{2}{\pi} \ln \left(2^{n-1}\right)+1\right)\left(\frac{2}{\pi} \ln \left(2^{t-2}\right)+1\right)$.

Доказательство. Согласно теореме 1 достаточно проверить справедливость оценки $\sigma(f) \leqslant \sigma_{n} \sigma_{t-1}$. Из теоремы 1 и равенства (10) следует, что при $t=2$ доказываемая оценка справедлива. Пусть теперь $t \geqslant 3$, тогда в силу соотношения (10)

$$
\sigma(f)=\frac{1}{2^{n-1}} \sum_{j \in\left\{1,3, \ldots, 2^{n}-1\right\}} \frac{c_{t j}}{\sin \frac{\pi j}{2^{n}}} .
$$

Отсюда следует, что

$$
\sigma(f)=\frac{1}{2^{n-1}} \sum_{j \in\left\{1,3, \ldots, 2^{n}-1\right\}} \frac{\sigma_{t-1}}{\sin \frac{\pi j}{2^{n}}}+\frac{R}{2^{n-1}},
$$

где

$$
R=\sum_{j \in\left\{1,3, \ldots, 2^{n}-1\right\}} \frac{c_{t j}-\sigma_{t-1}}{\sin \frac{\pi j}{2^{n}}} .
$$


Для доказательства неравенства (11) достаточно проверить, что $R \leqslant 0$. С использованием п. 1 утверждения 1 получим

$$
R=\sum_{k=0}^{2^{n-t}-1} \sum_{j \in\left\{1,3, \ldots, 2^{t}-1\right\}} \frac{c_{t j}-\sigma_{t-1}}{\sin \frac{\pi\left(j+2^{t} k\right)}{2^{n}}}
$$

Пусть

$$
R_{k}=\sum_{j \in\left\{1,3, \ldots, 2^{t}-1\right\}} \frac{c_{t j}-\sigma_{t-1}}{\sin \frac{\pi\left(j+2^{t} k\right)}{2^{n}}} .
$$

Неравенство $R \leqslant 0$ будет обосновано, если доказать, что $R_{k} \leqslant 0$ для всех $k=0,1, \ldots, 2^{n-t}-1$. Пусть

$$
h_{k}(x)=\frac{1}{\sin \frac{\pi\left(x+2^{t} k\right)}{2^{n}}} .
$$

Ввиду выбора параметров выполнено условие $x+2^{t} k \leqslant 2^{n}-1$, поэтому функция $h_{k}(x)$ выпукла вниз на отрезке $\left[1,2^{t}-1\right]$. С использованием п. 2 утверждения 1 получим

$$
R_{k}=\sum_{j \in\left\{1,3, \ldots, 2^{t}-1\right\}}\left(c_{t 2^{t-1}-j}-\sigma_{t-1}\right)\left(h_{k}\left(2^{t-1}-j\right)+h_{k}\left(2^{t-1}+j\right)\right) .
$$

Если число $s$ подобрано так, как в п. 5 утверждения 1, то рассмотрим представление $R_{k}=R_{k}^{(1)}+R_{k}^{(2)}$, где

$$
\begin{aligned}
R_{k}^{(1)} & =\sum_{j \in\{1,3, \ldots, s\}}\left(c_{t 2^{t-1}-j}-\sigma_{t-1}\right)\left(h_{k}\left(2^{t-1}-j\right)+h_{k}\left(2^{t-1}+j\right)\right), \\
R_{k}^{(2)} & =\sum_{j \in\left\{s+2, s+4, \ldots, 2^{t}-1\right\}}\left(c_{t 2^{t-1}-j}-\sigma_{t-1}\right)\left(h_{k}\left(2^{t-1}-j\right)+h_{k}\left(2^{t-1}+j\right)\right) .
\end{aligned}
$$

Так как $c_{t 2^{t-1}-j}-\sigma_{t-1}>0$ для всех $j=1,3, \ldots, s$, а $c_{t 2^{t-1}-j}-\sigma_{t-1} \leqslant 0$ для всех $j=s+2, s+4, \ldots, 2^{t-1}-1$, то по лемме 3 при всех $j=1,3, \ldots, 2^{t-1}-1$ верно неравенство

$$
\begin{aligned}
& \left(c_{t 2^{t-1}-j}-\sigma_{t-1}\right)\left(h_{k}\left(2^{t-1}-j\right)+h_{k}\left(2^{t-1}+j\right)\right) \leqslant \\
& \leqslant\left(c_{t 2^{t-1}-j}-\sigma_{t-1}\right)\left(h_{k}\left(2^{t-1}-s\right)+h_{k}\left(2^{t-1}+s\right)\right) .
\end{aligned}
$$


Значит,

$$
\begin{aligned}
& R_{k}^{(1)} \leqslant\left(h_{k}\left(2^{t-1}-s\right)+h_{k}\left(2^{t-1}+s\right)\right) \sum_{j \in\{1,3, \ldots, s\}}\left(c_{t 2^{t-1}-j}-\sigma_{t-1}\right) \\
& R_{k}^{(2)} \leqslant\left(h_{k}\left(2^{t-1}-s\right)+h_{k}\left(2^{t-1}+s\right)\right) \sum_{j \in\left\{s+2, s+4, \ldots, 2^{t}-1\right\}}\left(c_{t 2^{t-1}-j}-\sigma_{t-1}\right) .
\end{aligned}
$$

Согласно п. 4 утверждения 1, если

$$
a=\sum_{j \in\{1,3, \ldots, s\}}\left(c_{t 2^{t-1}-j}-\sigma_{t-1}\right),
$$

то

$$
\begin{aligned}
a+ & \sum_{j \in\left\{s+2, s+4, \ldots, 2^{t}-1\right\}}\left(c_{t 2^{t-1}-j}-\sigma_{t-1}\right)= \\
= & \sum_{j \in\left\{1,3, \ldots, 2^{t}-1\right\}} c_{t 2^{t-1}-j}-2^{t-2} \sigma_{t-1}=0
\end{aligned}
$$

значит,

$$
\sum_{j \in\left\{s+2, s+4, \ldots, 2^{t}-1\right\}}\left(c_{t 2^{t-1}-j}-\sigma_{t-1}\right)=-a
$$

и в силу неравенств (16), (17)

$$
\begin{aligned}
& R_{k}^{(1)} \leqslant a\left(h_{k}\left(2^{t-1}-s\right)+h_{k}\left(2^{t-1}+s\right)\right), \\
& R_{k}^{(2)} \leqslant-a\left(h_{k}\left(2^{t-1}-s\right)+h_{k}\left(2^{t-1}+s\right)\right) .
\end{aligned}
$$

Таким образом, $R_{k}=R_{k}^{(1)}+R_{k}^{(2)} \leqslant 0$.

Непосредственно из неравенства (6) и утверждения 2 получим основной результат этого параграфа.

Теорема 3. Если $f(a)=a_{n-1} \oplus a_{n-t}$, где $2 \leqslant t \leqslant n$, то для булевой функции $g$, построенной по правилу (2),

$$
\mathrm{nl}(g) \geqslant 2^{m-1}-\left(2^{n-1}-1\right) 2^{m / 2-1}\left(\frac{2}{\pi} \ln \left(2^{n-1}\right)+1\right)\left(\frac{2}{\pi} \ln \left(2^{t-2}\right)+1\right) .
$$


4. Функция $f(a)=a_{n-1} \oplus a_{n-t+1} \oplus a_{n-t}$

Рассмотрим отображение $f(a)=a_{n-1} \oplus a_{n-t+1} \oplus a_{n-t}$, в котором $3 \leqslant t \leqslant n, a=a_{0}+2 a_{1}+\ldots+2^{n-1} a_{n-1} \in \mathbb{Z}_{2^{n}}, a_{0}, a_{1}, \ldots, a_{n-1} \in\{0,1\}$. По теореме 2 для спектральных коэффициентов $\nu_{j}=\nu_{j}(f)$ справедливо соотношение

$$
\left|\nu_{j}\right|= \begin{cases}0, & \text { если } j-\text { четное число, } \\ \frac{1}{2^{n-1}} \frac{1}{\sin \frac{\pi j}{2^{n}}}\left|\operatorname{tg} \frac{\pi j}{2^{t}}\right|\left|\operatorname{tg} \frac{\pi j}{2^{t-1}}\right|, & \text { если } j-\text { нечетное число. }\end{cases}
$$

Утверждение 3. Пусть $d_{t j}=\left|\operatorname{tg} \frac{\pi j}{2^{t}}\right|\left|\operatorname{tg} \frac{\pi j}{2^{t-1}}\right|$, где $j=1,3, \ldots, 2^{n}-1$. Тогда при всех $t \geqslant 4$ :

1) $d_{t j}=d_{t 2^{t}+j}$ для всех таких $j$, что $2^{t}+j \leqslant 2^{n}-1$,

2) $d_{t j}=d_{t 2^{t}-j}$ для всех $j=1,3, \ldots, 2^{t}-1$,

3) справедливо равенство

$$
d_{t j}=\left|\frac{1}{\cos \frac{\pi j}{2^{t-1}}}-1\right|, \quad j=1,3, \ldots, 2^{n}-1,
$$

4) при $j=1,3, \ldots, 2^{t-2}-1$

$$
d_{t j}=\frac{1}{\cos \frac{\pi j}{2^{t-1}}}-1,
$$

5) $d_{t 2^{t-1}-j}=d_{t 2^{t-1}+j}$ для всех $j=1,3, \ldots, 2^{t-1}-1$,

6) $d_{t j}=d_{t 2^{t}-j}$ для всех $j=1,3, \ldots, 2^{t}-1$,

7) $d_{t 2^{t-1}-j}=d_{t j}+2$ для всех $j=1,3, \ldots, 2^{t-2}-1$,

8) справедливо равенство

$$
\frac{1}{2^{t-3}} \sum_{j \in\left\{1,3, \ldots, 2^{t-2}-1\right\}}\left(d_{t j}+1\right)=\sigma_{t-1},
$$

где величина $\sigma_{t-1}$ определена равенством (7),

9) числа $d_{t j}$ монотонно возрастают при всех $j \leqslant 2^{t-2}-1$,

10) существует такое единственное число $s \in\left\{3,5, \ldots, 2^{t-2}-1\right\}$, что

$$
\begin{aligned}
d_{t 1}+1<\sigma_{t-1}, \ldots, d_{t 2^{t-2}-s+2}+1 \leqslant \sigma_{t-1} & \\
& d_{t 2^{t-2}-s+1}+1>\sigma_{t-1}, \ldots, d_{t 2^{t-2}-1}+1>\sigma_{t-1} .
\end{aligned}
$$

2016, T. 7, № 3, C. 29-46 
Доказательство. Утверждения пп. 1, 2 и 3 очевидны. Утверждения пп. 4, 5, 6 и 7 следуют из п. 3. Докажем п. 8. Согласно п. 4

$$
\frac{1}{2^{t-3}} \sum_{j \in\left\{1,3, \ldots, 2^{t-2}-1\right\}}\left(d_{t j}+1\right)=\frac{1}{2^{t-3}} \sum_{j \in\left\{1,3, \ldots, 2^{t-2}-1\right\}} \frac{1}{\cos \frac{\pi j}{2^{t-1}}},
$$

тогда

$$
\begin{aligned}
& \frac{1}{2^{t-3}} \sum_{j \in\left\{1,3, \ldots, 2^{t-2}-1\right\}}\left(d_{t j}+1\right)= \\
& =\frac{1}{2^{t-3}} \sum_{j \in\left\{1,3, \ldots, 2^{t-2}-1\right\}} \frac{1}{\sin \frac{\pi\left(2^{t-2}-j\right)}{2^{t-1}}}=\sigma_{t-1} .
\end{aligned}
$$

Утверждение п. 9 следует из п. 4 , а п. 10 следует из пп. 8 и 9.

Утверждение 4. Если $f(a)=a_{n-1} \oplus a_{n-t+1} \oplus a_{n-t}$, где $3 \leqslant t \leqslant n$, то

$$
\sigma(f)=\sum_{j=0}^{2^{n}-1}\left|\nu_{j}\right| \leqslant \sigma_{n} \sigma_{t-1}
$$

В частности,

$$
\sigma(f) \leqslant\left(\frac{2}{\pi} \ln \left(2^{n-1}\right)+1\right)\left(\frac{2}{\pi} \ln \left(2^{t-2}\right)+1\right) .
$$

Доказательство. Согласно теореме 1 достаточно доказать справедливость оценки $\sigma(f) \leqslant \sigma_{n} \sigma_{t-1}$. Из утверждения 2 и равенства (18) следует, что при $t=3$ доказываемая оценка справедлива. Пусть теперь $t \geqslant 4$, тогда в силу соотношения (18)

$$
\sigma(f)=\frac{1}{2^{n-1}} \sum_{j \in\left\{1,3, \ldots, 2^{n}-1\right\}} \frac{d_{t j}}{\sin \frac{\pi j}{2^{n}}}
$$

и согласно п. 1 утверждения 3

$$
\sigma(f)=\frac{1}{2^{n-1}} \sum_{k=0}^{2^{n-t}-1} \sum_{j=1,3, \ldots, 2^{t}-1} \sum_{j \in\left\{1,3, \ldots, 2^{t}-1\right\}} \frac{d_{t j}}{\sin \frac{\pi\left(j+2^{t} k\right)}{2^{n}}} .
$$


С использованием равенства (15) получим

$$
\sigma(f)=\frac{1}{2^{n-1}} \sum_{k=0}^{2^{n-t}-1} Q_{k}
$$

где

$$
Q_{k}=\sum_{j \in\left\{1,3, \ldots, 2^{t}-1\right\}} d_{t j} h_{k}(j), \quad k=0,1, \ldots, 2^{n-t}-1
$$

Используя пп. 5, 6 и 7 утверждения 3, находим, что

$$
\begin{aligned}
Q_{k}= & \sum_{j \in\left\{1,3, \ldots, 2^{t-2}-1\right\}}\left(d_{t j} h_{k}(j)+d_{t 2^{t-1}-j} h_{k}\left(2^{t-1}-j\right)+\right. \\
& \left.+d_{t 2^{t-1}+j} h_{k}\left(2^{t-1}+j\right)+d_{t 2^{t}-j} h_{k}\left(2^{t}-j\right)\right)= \\
=\sum_{j \in\left\{1,3, \ldots, 2^{t-2}-1\right\}}( & d_{t j}\left(h_{k}(j)+h_{k}\left(2^{t}-j\right)\right)+ \\
& \left.+\left(d_{t j}+2\right)\left(h_{k}\left(2^{t-1}-j\right)+h_{k}\left(2^{t-1}+j\right)\right)\right) .
\end{aligned}
$$

Запишем величину $Q_{k}$ следующим образом:

$$
\begin{aligned}
Q_{k}= & \sum_{j \in\left\{1,3, \ldots, 2^{t-2}-1\right\}}\left(d_{t j}+1\right)\left(h_{k}(j)+h_{k}\left(2^{t-1}-j\right)+\right. \\
& \left.+h_{k}\left(2^{t-1}+j\right)+h_{k}\left(2^{t}-j\right)\right)+ \\
& +\sum_{j \in\left\{1,3, \ldots, 2^{t-2}-1\right\}}\left(-h_{k}(j)+h_{k}\left(2^{t-1}-j\right)+h_{k}\left(2^{t-1}+j\right)-h_{k}\left(2^{t}-j\right)\right) .
\end{aligned}
$$


В силу выпуклости вниз функции $h_{k}(x)$ на отрезке $\left[1,2^{t}-1\right]$ и леммы 2

$$
-h_{k}(j)+h_{k}\left(2^{t-1}-j\right)+h_{k}\left(2^{t-1}+j\right)-h_{k}\left(2^{t}-j\right) \leqslant 0,
$$

поэтому

$$
\begin{aligned}
Q_{k} \leqslant \sum_{j \in\left\{1,3, \ldots, 2^{t-2}-1\right\}}\left(d_{t j}+1\right)( & h_{k}(j)+h_{k}\left(2^{t-1}-j\right)+ \\
& \left.+h_{k}\left(2^{t-1}+j\right)+h_{k}\left(2^{t}-j\right)\right) .
\end{aligned}
$$

Из равенства (20) следует, что

$$
\begin{aligned}
\sigma(f) \leqslant \frac{1}{2^{n-1}} \sum_{k=0}^{2^{n-t}-1} \sum_{j \in\left\{1,3, \ldots, 2^{t-2}-1\right\}}\left(d_{t j}+1\right)\left(h_{k}(j)+h_{k}\left(2^{t-1}-j\right)+\right. \\
\left.+h_{k}\left(2^{t-1}+j\right)+h_{k}\left(2^{t}-j\right)\right)= \\
=\frac{\sigma_{t-1}}{2^{n-1}} \sum_{k=0}^{2^{n-t}-1} \sum_{j \in\left\{1,3, \ldots, 2^{t-2}-1\right\}}\left(h_{k}(j)+h_{k}\left(2^{t-1}-j\right)+\right. \\
\left.+h_{k}\left(2^{t-1}+j\right)+h_{k}\left(2^{t}-j\right)\right)+\frac{D}{2^{n-1}}= \\
=\sigma_{t-1} \sigma_{n}+\frac{D}{2^{n-1}},
\end{aligned}
$$

где

$$
D=\sum_{k=0}^{2^{n-t}-1}\left(D_{k}^{(1)}+D_{k}^{(2)}\right)
$$

a

$$
\begin{aligned}
D_{k}^{(1)} & =\sum_{j \in\left\{1,3, \ldots, 2^{t-2}-1\right\}}\left(d_{t j}+1-\sigma_{t-1}\right)\left(h_{k}(j)+h_{k}\left(2^{t}-j\right)\right), \\
D_{k}^{(2)} & =\sum_{j \in\left\{1,3, \ldots, 2^{t-2}-1\right\}}\left(d_{t j}+1-\sigma_{t-1}\right)\left(h_{k}\left(2^{t-1}-j\right)+h_{k}\left(2^{t-1}+j\right)\right) .
\end{aligned}
$$

Докажем, что при всех $k=0,1, \ldots, 2^{n-t}-1$ выполнены неравенства

$$
D_{k}^{(1)} \leqslant 0, \quad D_{k}^{(2)} \leqslant 0 .
$$

Этим будет доказано, что $D \leqslant 0$, а значит, $\sigma(f) \leqslant \sigma_{t-1} \sigma_{n}$. 
Используя пп. 8, 10 утверждения 3 и лемму 2, получаем:

$$
\begin{aligned}
D_{k}^{(1)}= & \sum_{j \in\left\{1,3, \ldots, 2^{t-2}-s\right\}}\left(d_{t j}+1-\sigma_{t-1}\right)\left(h_{k}(j)+h_{k}\left(2^{t}-j\right)\right)+ \\
& +\sum_{j \in\left\{2^{t-2}-s+2, \ldots, 2^{t-2}-1\right\}}\left(d_{t j}+1-\sigma_{t-1}\right)\left(h_{k}(j)+h_{k}\left(2^{t}-j\right)\right) \leqslant \\
\leqslant & \sum_{j \in\left\{1,3, \ldots, 2^{t-2}-s\right\}}\left(d_{t j}+1-\sigma_{t-1}\right)\left(h_{k}\left(2^{t-2}-s\right)+h_{k}\left(2^{t}-2^{t-2}+s\right)\right)+ \\
& +\sum_{j \in\left\{2^{t-2}-s+2, \ldots, 2^{t-2}-1\right\}}\left(d_{t j}+1-\sigma_{t-1}\right)\left(h_{k}\left(2^{t-2}-s\right)+h_{k}\left(2^{t}-2^{t-2}+s\right)\right)= \\
= & \left(h_{k}\left(2^{t-2}-s\right)+h_{k}\left(2^{t}-2^{t-2}+s\right)\right) \sum_{j \in\left\{1,3, \ldots, 2^{t-2}-1\right\}}\left(d_{t j}+1-\sigma_{t-1}\right)=0 .
\end{aligned}
$$

Аналогично доказывается, что $D_{k}^{(2)} \leqslant 0$.

Из неравенства (6) и утверждения 4 вытекает следующий результат.

Теорема 4. Если

$$
f(a)=a_{n-1} \oplus a_{n-t+1} \oplus a_{n-t},
$$

где $3 \leqslant t \leqslant n$, то для булевой функции $g$, построенной по правилу (2),

$$
\mathrm{nl}(g) \geqslant 2^{m-1}-\left(2^{n-1}-1\right) 2^{m / 2-1}\left(\frac{2}{\pi} \ln \left(2^{n-1}\right)+1\right)\left(\frac{2}{\pi} \ln \left(2^{t-2}\right)+1\right) .
$$

\section{5. Функции, линейные относительно старших четырех битов}

В этом параграфе рассматриваются функции $f$, задаваемые выражением $f(a)=a_{n-1} \oplus c_{1} a_{n-2} \oplus c_{2} a_{n-3} \oplus c_{3} a_{n-4}$, где $c_{1}, c_{2}, c_{3} \in\{0,1\}$ суть фиксированные элементы. 
Утверждение 5. Пусть $\sigma_{n}$ - величина, заданная выражениями (7). Тогда:

1) если $f(a)=a_{n-1}$ или $f(a)=a_{n-1} \oplus a_{n-2}$, то

$$
\sigma(f) \leqslant \sigma_{n} \leqslant \frac{2}{\pi} \ln \left(2^{n-1}\right)+1,
$$

2) если $f(a)=a_{n-1} \oplus a_{n-2} \oplus a_{n-3}$ или $f(a)=a_{n-1} \oplus a_{n-3}$, то

$$
\sigma(f) \leqslant \sqrt{2} \sigma_{n} \leqslant \sqrt{2}\left(\frac{2}{\pi} \ln \left(2^{n-1}\right)+1\right)
$$

3) если $f(a)=a_{n-1} \oplus a_{n-4}$ или $f(a)=a_{n-1} \oplus a_{n-3} \oplus a_{n-4}$, или $f(a)=$ $a_{n-1} \oplus a_{n-2} \oplus a_{n-4}$, или $f(a)=a_{n-1} \oplus a_{n-2} \oplus a_{n-3} \oplus a_{n-4}$, то

$$
\sigma(f) \leqslant \sqrt{2+\sqrt{2}} \sigma_{n} \leqslant \sqrt{2+\sqrt{2}}\left(\frac{2}{\pi} \ln \left(2^{n-1}\right)+1\right)
$$

Доказательство. 1) Используя теорему 2 и равенство $\left|\operatorname{tg} \frac{\pi j}{4}\right|=1, \quad j=$ $1,3, \ldots, 2^{n}-1$, получим

$$
\left|\nu_{j}\right|= \begin{cases}0, & \text { если } j-\text { четное число, } \\ \frac{1}{2^{n-1} \frac{1}{\sin \frac{\pi j}{2^{n}}},} & \text { если } j-\text { нечетное число. }\end{cases}
$$

Остается воспользоваться теоремой 1.

2) Согласно теореме 2 в этом случае

$$
\left|\nu_{j}\right|= \begin{cases}0, & \text { если } j-\text { четное число, } \\ \frac{1}{2^{n-1}} \frac{1}{\sin \frac{\pi j}{2^{n}}}\left|\operatorname{tg} \frac{\pi j}{8}\right|, & \text { если } j-\text { нечетное число. }\end{cases}
$$

По утверждению 2 имеем $\sigma(f) \leqslant \sigma_{2} \sigma_{n}$. Остается заметить, что $\sigma_{2}=\sqrt{2}$. 
3) Применяя теорему 2 к отображениям $f(a)=a_{n-1} \oplus a_{n-2} \oplus a_{n-4}$ и $f(a)=a_{n-1} \oplus a_{n-4}$, получим

$$
\left|\nu_{j}\right|= \begin{cases}0, & \text { если } j-\text { четное число, } \\ \frac{1}{2^{n-1}} \frac{1}{\sin \frac{\pi j}{2^{n}}}\left|\operatorname{tg} \frac{\pi j}{16}\right|, & \text { если } j-\text { нечетное число. }\end{cases}
$$

Аналогично, для отображений $f(a)=a_{n-1} \oplus a_{n-2} \oplus a_{n-3} \oplus a_{n-4}$ и $f(a)=a_{n-1} \oplus a_{n-3} \oplus a_{n-4}$ получаем

$$
\left|\nu_{j}\right|= \begin{cases}0, & \text { если } j-\text { четное число, } \\ \frac{1}{2^{n-1}} \frac{1}{\sin \frac{\pi j}{2^{n}}}\left|\operatorname{tg} \frac{\pi j}{16}\right|\left|\operatorname{tg} \frac{\pi j}{8}\right|, & \text { если } j-\text { нечетное число. }\end{cases}
$$

Согласно утверждениям 2 и 4 в этих случаях $\sigma(f) \leqslant \sigma_{3} \sigma_{n}$. Остается заметить, что $\sigma_{3}=\sqrt{2+\sqrt{2}}$.

Таким образом, неравенство (6) и утверждение 5 обосновывают следующую теорему.

Теорема 5. Пусть булева функиия g построена по правилу (2). Тогда:

1) если $f(a)=a_{n-1}$ или $f(a)=a_{n-1} \oplus a_{n-2}$, то

$$
\operatorname{nl}(g) \geqslant 2^{m-1}-\left(2^{n-1}-1\right) 2^{m / 2-1}\left(\frac{2}{\pi} \ln \left(2^{n-1}\right)+1\right),
$$

2) если $f(a)=a_{n-1} \oplus a_{n-2} \oplus a_{n-3}$ или $f(a)=a_{n-1} \oplus a_{n-3}$, то

$$
\mathrm{nl}(g) \geqslant 2^{m-1}-\left(2^{n-1}-1\right) 2^{m / 2-1} \sqrt{2}\left(\frac{2}{\pi} \ln \left(2^{n-1}\right)+1\right),
$$

3) если $f(a)=a_{n-1} \oplus a_{n-4}$ или $f(a)=a_{n-1} \oplus a_{n-3} \oplus a_{n-4}$, или $f(a)=$ $a_{n-1} \oplus a_{n-2} \oplus a_{n-4}$, или $f(a)=a_{n-1} \oplus a_{n-2} \oplus a_{n-3} \oplus a_{n-4}$, то

$$
\mathrm{nl}(g) \geqslant 2^{m-1}-\left(2^{n-1}-1\right) 2^{m / 2-1} \sqrt{2+\sqrt{2}}\left(\frac{2}{\pi} \ln \left(2^{n-1}\right)+1\right) .
$$




\section{Список литературы}

[1] Глухов М. М., Елизаров В. П., Нечаев А. А., Алгебра, 2, М. : Гелиос АРВ, 2003, 416 с.

[2] Лидл Р., Нидеррайтер Г., Конечные поля, М.: Мир, 1988, 824 с.

[3] Нечаев А. А., "Цикловые типы линейных подстановок над конечными коммутативными кольцами", Матем. сборник, $184: 3$ (1993), 21-56.

[4] Логачев О.А., Сальников А. А., Ященко В.В., Булевы функиии в теории кодирования и криптологии, М.: МЦНМО, 2012, 584 с.

[5] Камловский О. В., "Расстояние между двоичными представлениями линейных рекуррент над полем $G F\left(2^{k}\right)$ и кольцом $\mathbb{Z}_{2^{n}}$ ", Математические вопросы криптографии, 7:1 (2016), 71-82.

[6] Былков Д.Н., “Об одном классе булевых функций, построенных с использованием старших разрядных последовательностей линейных рекуррент”, Прикладная дискретная математика. Приложение, № 7 (2014), 59-60.

[7] Shparlinski I. E., Winterhof A., "On the nonlinearity of linear recurrence sequences.", Applied Math. Letters, 19:4 (2006), 340-344.

[8] Амбросимов А.С., “Свойства бент-функций q-значной логики над конечными полями”, Дискретная математика, $6: 3$ (1994), 50-60.

[9] Солодовников В.И., “Бент-функции из конечной абелевой группы в конечную абелеву группу”, Дискретная математика, 14:1 (2002), 99-113.

[10] Камловский О.В., "Метод тригонометрических сумм для исследования частот $r$-грамм в старших координатных последовательностях линейных рекуррент над кольцом $\mathbb{Z}_{2}{ }^{n}$ ”, Математические вопросы криптографии, 1:4 (2010), 33-62.

[11] Камловский О. В., "Равномерные последовательности над простыми полями, построенные из одного класса линейных рекуррент над кольцами вычетов”, Проблемы передачи информачии, 50:2 (2014), 60-76.

[12] Камловский О. В., “Частотные характеристики разрядных последовательостей линейных рекуррент над кольцами Галуа”, Изв. РАН, 77 :6 (2013), 71-96. 